Research Paper

\title{
The Hemoglobin, Albumin, Lymphocyte, and Platelet (HALP) Score is a Novel Significant Prognostic Factor for Patients with Metastatic Prostate Cancer Undergoing Cytoreductive Radical Prostatectomy
}

Yadong Guo ${ }^{*}$, Donghui Shi ${ }^{*}$, Junfeng Zhang ${ }^{1 *}$, Shiyu Mao ${ }^{1}$, Longsheng Wang ${ }^{1}$, Wentao Zhang 3 , Ziwei

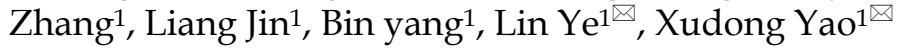

1. Department of Urology, Shanghai Tenth People's Hospital, Tongji University, Shanghai, P.R. China

2. Department of Urology, Wuzhong People's Hospital of Suzhou, Jiangsu, P.R. China

3. Department of Anhui Medical University, Hefei, P.R. China

* These authors contributed equally to this study.

$\square$ Corresponding authors: Lin Ye, E-mail: ericyelin@163.com; Xudong Yao, E-mail: yaoxudong078@sina.com. Department of Urology, Shanghai Tenth People’s Hospital, Tongji University. 301 Yanzhong Road, Jing'an District, Shanghai 200040, Tel: +86 02166301073.

(C) Ivyspring International Publisher. This is an open access article distributed under the terms of the Creative Commons Attribution (CC BY-NC) license (https://creativecommons.org/licenses/by-nc/4.0/). See http://ivyspring.com/terms for full terms and conditions.

Received: 2018.05.11; Accepted: 2018.10.01; Published: 2019.01.01

\begin{abstract}
Objective: The hemoglobin, albumin, lymphocyte, and platelet (HALP) score has been shown to be an important prognostic marker in some tumor types. The aim of this study was to evaluate the prognostic impact of the preoperative HALP score, with the intent to develop a new prognostic index for patients with metastatic prostate cancer (mPCA) after cytoreductive radical prostatectomy (cRP).

Methods: We retrospectively analyzed the data from 82 patients with mPCA after cRP in our institution. Of these patients, 70 patients were diagnosed with oligometastatic prostate cancer (oPCA). The main outcome measure was prostate-specific antigen (PSA) progression-free survival (PFS), which was assessed using Kaplan-Meier curves with log-rank statistics. In addition, univariate and multivariate Cox regression analyses were performed to determine the prognostic factors associated with PSA-PFS. The prediction accuracy was evaluated by assessing the area under the receiver operating characteristic (AUC) curve.

Results: The median follow-up time for all patients was 17.47 months (range: 11.73-24.38 months). Based on the Kaplan-Meier curve analysis, it was noticed that a low preoperative HALP value $(<32.4)$ was significantly associated with a decreased PSA-PFS in both the mPCA and oPCA subgroups $(P<0.001, P=$ 0.002 , respectively). In addition, multivariate analysis predicted that a low HALP score was a common independent prognostic factor of an overall shorter PSA-PFS (HR: 0.352; range: 0.154-0.804; $P=0.013$ ). However, among the different subgroups, a low HALP score (HR: 0.275 ; range: $0.116-0.653 ; P=0.003$ ) was confirmed to be an independent predictor of a shorter PSA-PFS in patients from the oPCA subgroup. Furthermore, the effective combination of the pathologic Gleason score (PGS) and the HALP score (HALPG) as a new index was found to be an independent risk factor. Also, the AUC of the HALPG score for PSA-PFS was observed to be higher than other conventional clinical indices.

Conclusion: Overall, our results confirmed the HALP score as an independent prognostic factor for PSA-PFS in patients with mPCA or oPCA after cRP. Moreover, the new index, HALPG, also appeared to be an independent prognostic factor and was better than the HALP score. Importantly, it is evident that this new prognostic index has the ability to accurately identify patients at low, intermediate, and high risk of recurrence, thus easily allowing informed treatment decisions to be made.
\end{abstract}

Key words: metastatic prostate cancer, prognostic model, hemoglobin, albumin, lymphocyte, and platelet (HALP) score 


\section{Introduction}

Prostate cancer is the most prevalent malignancy affecting men in the USA, with an estimated 164,690 new diagnoses and 29,430 deaths expected this year [1]. Androgen deprivation therapy (ADT) has long been used as a cornerstone for the treatment of prostate cancer, especially local high-risk and metastatic prostate cancer (mPCA) $[2,3]$. Recently, with the use of additional innovative approaches, efforts have been made to improve the prognosis and quality of life of these cancer patients. For instance, cytoreductive radical prostatectomy (cRP) in patients with mPCA, especially in those with oligometastatic prostate cancer (oPCA), which is defined as the transitional state between localized disease and its widespread metastasis as well as the number of metastases $\leq 5$, has been performed[4-6]. Typically, mPCA is a high-risk, fatal disease, with a varied prognosis; for example, a 5 -year survival of $100 \%$ for patients with localized disease has been reported, while it was only $28 \%$ in patients diagnosed with distant metastasized disease [7, 8]. Therefore, it is essential to find biomarkers that can guide and help to stratify mPCA patients for specific treatment decisions and predicting the prognosis.

Cancer progression and its metastasis are not only dependent on the type of tumor cells, but nutrition and immunity also play an important role in these processes [9]. Inflammatory cells and immune responses have consistently been recognized as important factors in the prognosis of cancer [10,11]. Previous studies have reported that peripheral blood cells, including neutrophils, lymphocytes, platelets, and monocytes, can promote tumor proliferation, invasion, and metastasis $[12,13]$. Thus, based on this evidence, many combinations of inflammatory indices such as the neutrophil-to-lymphocyte ratio (NLR), platelet-to-lymphocyte ratio (PLR), and the systemic immune-inflammation index [(platelet $\times$ neutrophil $) /$ lymphocyte] have been used to predict the PCA prognosis [14-16]. In parallel, the nutritional status of cancer patients also has been indicated as an important parameter affecting survival outcomes, including hemoglobin, albumin levels, and other nutrition indices [17-20]. Recent studies have identified a new inflammation index called HALP, comprised of hemoglobin, albumin, lymphocytes, and platelets, which has proven to be a good prognostic indicator in gastric, colorectal, renal, and bladder cancers [21-24]. However, to the best of our knowledge, no studies have been performed to assess the HALP score as a predictive marker of prognosis for patients diagnosed with mPCA. Thus, the aim of this study was to investigate the prognostic role of the HALP score and also to develop a new prognostic model for mPCA patients after cRP.

\section{Materials and Methods}

\section{Patient selection}

This current study was approved by the Institutional Review Board of Shanghai Tenth People's Hospital, and all patients provided written informed consent. A total of 82 patients with mPCA who underwent surgery at the Urology Department, Shanghai Tenth People's Hospital, between 2013 and 2017, were included in our analyses. The clinical and pathological features mainly included age, serum prostate-specific antigen (PSA) levels, blood cell counts, and pathologic Gleason score (PGS). All blood samples were collected within a week prior to surgery, and patients underwent computed tomography, magnetic resonance imaging, emission computed tomography, and pathological biopsy before surgery for mPCA through a multidisciplinary team discussion. The postoperative pathological results were reported as adenocarcinoma. The patients were excluded from our study if there was incomplete clinical data, there was no follow-up, they did not have a cRP, or they had a nonmetastatic prostate cancer diagnosis.

\section{Follow-up and evaluation}

The patients were followed up after the cRP every 3 months for up to 2 years, then every 6 months for another 3 years, and yearly thereafter. Postoperative investigations primarily included measurement of the serum concentrations of PSA, testosterone, glucose, and alkaline phosphatase. Missing survival data were obtained by telephone contact. No routine imaging studies were performed, unless the serum PSA levels increased to more than $5 \mathrm{ng} / \mathrm{mL}$ or other clinical symptoms were identified. The study endpoints were PSA progression-free survival (PSA-PFS). Due to the small number of deaths, we could not evaluate overall survival as an end point. PSA progression was defined as three consecutive increases in PSA levels within 1 week apart, resulting in two $50 \%$ increases over the nadir, and PSA levels of $>2 \mathrm{ng} / \mathrm{mL}$. PSA-PFS was defined as the time from the initiation of ADT to the first evidence of biochemical progression. The HALP score was calculated as hemoglobin $(\mathrm{g} / \mathrm{L}) \times$ albumin $(\mathrm{g} / \mathrm{L})$ levels $\times$ lymphocyte count (/L)/platelet count (/L), with the platelet-lymphocyte ratio (PLR) as the platelet count divided by the lymphocyte count and the neutrophil-lymphocyte ratio (NLR) as the neutrophil count divided by the lymphocyte count.

\section{Statistical analysis}

X-tile software v3.6.1 (Yale University) was used 
to determine the optimal cutoff values of the NLR, PLR, and HALP score [25]; and SPSS v24.0 (SPSS Inc., Chicago, IL, USA) was used for all statistical analyses. The medians and interquartile ranges (IQRs) were assessed for continuous variables, while frequencies and proportions are reported for categorical variables. The association between the clinicopathological data and the HALP score was evaluated by the chi-squared and Mann-Whitney $U$ tests. The correlations with PSA-PFS were assessed using Kaplan-Meier curves with log-rank statistics. Furthermore, univariate and multivariate Cox regression analyses were used to calculate the respective hazard ratios (HRs) and 95\% confidence intervals (CIs). Only factors determined to be significant according to the univariate analyses were subsequently included in the multivariate analyses. Receiver operating characteristics (ROC) curves were used to define sensitivity and specificity, and the differences in the areas under the curve (AUC) were detected using MedCalc, version 15.2.0 (MedCalc software). For all analyses, a P value of < 0.05 was considered statistically significant.

\section{Results}

\section{Patient characteristics and the relationship between the HALP score and clinicopathological features}

Among the 82 mPCA patients who met the eligibility criteria between 2013 and 2017, 70 (85.4\%) patients with oPCA were included. The observed median age was 69 years old (range: 63-73 years old), while the median baseline PSA level was $75.05 \mathrm{ng} / \mathrm{dI}$ (23.93-100 ng/dI). At the time of enrollment, 39 (47.6\%) patients had lymph node metastasis, and 56 $(68.3 \%)$ had positive surgical margins. Among the PGS values available for all 82 patients, $65(79.3 \%)$ of them had a score of $8-10$. The median preoperative HALP value was 45.77 (range: 33.08-64.55). In addition, $46(56.1 \%)$ patients received neoadjuvant ADT before cRP. The median follow-up time was 17.47 months, and $5(6.1 \%)$ patients died during this period. The observed median PSA-PFS was 16.42 months (range: 8.81-24.03 months). All other clinicopathological characteristics are summarized in Table 1.

Based on the results obtained using X-tile software, the following cutoff values were observed: 32.4 for HALP, 191.8 for PLR, and 2.9 for NLR (Figure 1). Subsequently, 82 patients were divided into low-HALP $(\mathrm{n}=18,22 \%)$ and high-HALP $(\mathrm{n}=64,78 \%)$ groups. Moreover, the PGS, American Society of Anesthesiologists (ASA), NLR, and PLR were determined to be associated with a low HALP score (Table 2).
Table 1. Clinicopathological characteristics of 82 prostate cancer patients

\begin{tabular}{ll}
\hline Characteristic (n = 82) & Value \\
\hline Age, median (IQR), years & $69(63-73)$ \\
PSA, median (IQR), ng/mL & $75.05(27.93-100)$ \\
Body mass index & \\
$<24$ & $48(58.5)$ \\
$\geq 24$ & $34(41.5)$ \\
Diabetes & \\
yes & $16(19.5 \%)$ \\
no & $66(80.5 \%)$ \\
Hypertension & \\
yes & $35(42.7 \%)$ \\
no & $47(57.3 \%)$ \\
Pathologic Gleason Score & \\
6 & $3(3.7 \%)$ \\
7 & $14(17.1 \%)$ \\
8 & $15(18.3 \%)$ \\
9 & $47(57.3 \%)$ \\
10 & $3(3.7 \%)$ \\
pT-stage & \\
2 & $11(13.4 \%)$ \\
3 & $47(57.3 \%)$ \\
4 & $24(29.3 \%)$ \\
pN-stage & \\
negative & $43(52.4 \%)$ \\
positive & $39(47.6 \%)$ \\
Neoadjuvant ADT & \\
yes & $46(56.1 \%)$ \\
no & $36(43.9 \%)$ \\
Positive margins & \\
yes & $56(68.3 \%)$ \\
no & $26(31.7 \%)$ \\
ASA grade & \\
$1-2$ & $68(88.3 \%)$ \\
$3-4$ & $14(11.7 \%)$ \\
NLR, median (IQR) & $2.27(1.54-3.12)$ \\
PLR, median (IQR) & $119.05(85.72-166.92)$ \\
HALP, median (IQR) & $45.77(33.08-64.55)$ \\
PSA-PFS, median (IQR), months & $16.42(8.81-24.03)$ \\
Follow-up time, median (IQR), months & $17.47(11.73-24.38)$ \\
\hline PSA, prostate-specific antigen; IQR, interquartile range; pT-stage, pathological T \\
staging; pN-stage, pathological lymph node status; ADT, androgen-deprivation \\
therapy; ASA, American Society of Anesthesiologists; NLR: \\
neutrophil-to-lymphocyte ratio; PLR: platelet-to-lymphocyte ratio; HALP, \\
hemoglobin, albumin, lymphocyte, and platelet score; PSA-PF, prostate-specific \\
antigen progression-free survival. & \\
&
\end{tabular}

\section{Predicting the role of the HALP score in mPCA patients after $\mathbf{C R P}$}

Next, we observed that PSA progression occurred in 26 of the 82 patients (31.7\%); and among these, 12 were from the low-HALP group $(66.7 \%)$ and another 14 belonged to the high-HALP group (21.9\%). The patients in the high-HALP group had higher 2-year PSA-PFS rates than those in the low-HALP group $(72.3 \%$ vs. $26.7 \%)$. In addition, Kaplan-Meier analysis indicated that a low HALP score, along with high NLR and PLR values, was associated with a shorter PSA-PFS in mPCA (log-rank test $\mathrm{P}<0.001, \mathrm{P}=$ 0.001 , and $\mathrm{P}=0.019$, respectively) and oPCA $(\mathrm{P}=$ $0.002, \mathrm{P}=0.015$, and $\mathrm{P}=0.037$, respectively; Figure 2 ) patients. Also, the patients in the mPCA group showed age $(\mathrm{P}=0.037)$, $\mathrm{PGS}(\mathrm{P}=0.006), \mathrm{NLR}(\mathrm{P}=$ 
0.023), PLR ( $\mathrm{P}=0.002)$, and HALP score $(\mathrm{P}=0.001)$ as significant predictors in the prognosis of PSA-PFS, by univariate analysis. Similarly, in the oPCA subgroup, the HALP score $(\mathrm{P}=0.003)$ displayed important prognostic significance. Furthermore, multivariate analysis also indicated the HALP score $(\mathrm{P}=0.013)$ as an independent predictor of $\mathrm{MPCA}$, along with PGS $(P=0.038)$. Similarly, the HALP score was also confirmed as an independent prognostic factor in oPCA patients $(\mathrm{P}=0.003)$. Moreover, a low HALP ( $\mathrm{HR}=0.352,95 \% \mathrm{CI}: 0.154-0.804, \mathrm{P}=0.013)$ was only identified as a significant predictor for PSA-PFS of mPCA, while NLR and PLR did not show predictive value (Table 3 - Table 4). Overall, our data indicated that the preoperative HALP score is an independent predictor of PFS in patients with MPCA or oPCA after cRP.

Table 2. Association between clinicopathological characteristics and HALP values. Data are shown as $\mathrm{n}(\%)$

\begin{tabular}{|c|c|c|c|}
\hline \multirow[t]{2}{*}{ Cohort characteristics } & \multicolumn{3}{|l|}{ HALP value } \\
\hline & Low $(n=18)$ & High $(n=64)$ & Pvalue \\
\hline Age, years & & & 0.086 \\
\hline$<69$ & $12(66.7 \%)$ & $28(43.8 \%)$ & \\
\hline$\geq 69$ & $6(33.3 \%)$ & $36(56.3 \%)$ & \\
\hline $\mathrm{PSA}, \mathrm{ng} / \mathrm{ml}$ & & & 0.762 \\
\hline$\leq 20$ & $4(22.2 \%)$ & $10(15.6 \%)$ & \\
\hline$>20$ & $14(77.8 \%)$ & $54(84.4 \%)$ & \\
\hline BMI & & & 0.802 \\
\hline$<24$ & $11(61.1 \%)$ & $37(57.8 \%)$ & \\
\hline$\geq 24$ & $7(38.9 \%)$ & $27(42.2 \%)$ & \\
\hline diabetes & & & 0.181 \\
\hline yes & $6(33.3 \%)$ & $10(15.6 \%)$ & \\
\hline no & $12(66.7 \%)$ & $54(84.4 \%)$ & \\
\hline hypertension & & & 0.364 \\
\hline yes & $6(33.3 \%)$ & $29(45.3 \%)$ & \\
\hline no & $12(66.7 \%)$ & $35(54.7 \%)$ & \\
\hline Pathologic Gleason Score & & & 0.029 \\
\hline 6 & $1(5.6 \%)$ & $2(3.1 \%)$ & \\
\hline 7 & $1(5.6 \%)$ & $13(20.3 \%)$ & \\
\hline 8 & $2(11.1 \%)$ & $13(20.3 \%)$ & \\
\hline 9 & $11(61.1 \%)$ & $36(56.3 \%)$ & \\
\hline 10 & $3(16.7 \%)$ & $0(0 \%)$ & \\
\hline pT-stage & & & 0.676 \\
\hline 2 & $2(11.1 \%)$ & $9(14.1 \%)$ & \\
\hline 3 & $12(66.7 \%)$ & $35(54.7 \%)$ & \\
\hline 4 & $4(22.2 \%)$ & $20(31.3 \%)$ & \\
\hline pN-stage & & & 0.764 \\
\hline negative & $10(55.6 \%)$ & $33(51.6 \%)$ & \\
\hline positive & $8(44.4 \%)$ & $31(48.4 \%)$ & \\
\hline Neoadjuvant ADT & & & 0.628 \\
\hline yes & $11(61.1 \%)$ & $35(54.7 \%)$ & \\
\hline no & $7(38.9 \%)$ & $29(45.3 \%)$ & \\
\hline Positive margins & & & 0.867 \\
\hline yes & $12(66.7 \%)$ & $44(68.7 \%)$ & \\
\hline no & $6(33.3 \%)$ & $20(31.3 \%)$ & \\
\hline ASA grade & & & 0.002 \\
\hline $1 \& 2$ & $10(55.6 \%)$ & $58(90.6 \%)$ & \\
\hline $3 \& 4$ & $8(44.4 \%)$ & $6(9.4 \%)$ & \\
\hline NLR & & & $<0.001$ \\
\hline low & $3(16.7 \%)$ & $51(79.7 \%)$ & \\
\hline high & $15(83.3 \%)$ & $13(20.3 \%)$ & \\
\hline PLR & & & $<0.001$ \\
\hline
\end{tabular}

\begin{tabular}{|c|c|c|c|}
\hline \multirow[t]{2}{*}{ Cohort characteristics } & \multicolumn{3}{|l|}{ HALP value } \\
\hline & Low $(n=18)$ & High $(n=64)$ & Pvalue \\
\hline low & $5(27.8 \%)$ & $64(100 \%)$ & \\
\hline high & $13(72.2 \%)$ & $0(0 \%)$ & \\
\hline
\end{tabular}

PSA, prostate-specific antigen; $\mathrm{pT}$-stage, pathological T staging; $\mathrm{pN}$-stage, pathological lymph node status; ADT, androgen-deprivation therapy; ASA, American Society of Anesthesiologists; NLR: neutrophil-to-lymphocyte ratio; PLR: platelet-to-lymphocyte ratio; PSA-PFS, prostate-specific antigen progression-free survival.

Table 3. Univariate and multivariate analyses of factors associated with PSA-PFS in mPCA patients who had cytoreductive radical prostatectomy

\begin{tabular}{|c|c|c|c|c|}
\hline \multirow[t]{2}{*}{ Variable } & \multicolumn{2}{|l|}{ Univariate analysis } & \multicolumn{2}{|c|}{ Multivariate analysis } \\
\hline & HR (95\% CI) & Pvalue & HR ( $95 \%$ CI) & P value \\
\hline Age $(\geq 69 \mathrm{vs}<69)$ & $0.422(0.188-0.951)$ & 0.037 & - & - \\
\hline $\operatorname{PSA}(\geq 20 v s<20)$ & $1.745(0.614-4.964)$ & 0.296 & & \\
\hline $\operatorname{BMI}(\geq 24 \mathrm{vs}<24)$ & $1.019(0.464-2.237)$ & 0.963 & & \\
\hline diabetes & $1.317(0.551-3.147)$ & 0.536 & & \\
\hline hypertension & $1.266(0.585-2.739)$ & 0.55 & & \\
\hline $\begin{array}{l}\text { Pathologic Gleason } \\
\text { Score }\end{array}$ & $2.401(1.290-4.469)$ & 0.006 & $\begin{array}{l}1.858 \\
(1.035-3.335)\end{array}$ & 0.038 \\
\hline pT34vs2 & $1.185(0.667-2.106)$ & 0.562 & & \\
\hline pN-stage & $1.461(0.671-3.181)$ & 0.34 & & \\
\hline Positive margins & 0.791(0.351-1.783) & 0.572 & & \\
\hline $\begin{array}{l}\text { ASA grade(1\&2 vs } \\
3 \& 4)\end{array}$ & $1.233(0.494-3.078)$ & 0.653 & & \\
\hline Neoadjuvant ADT & $1.298(0.578-2.918)$ & 0.527 & & \\
\hline $\operatorname{NLR}(\geq 2.9 v s<2.9)$ & $2.452(1.131-5.318)$ & 0.023 & - & - \\
\hline $\operatorname{PLR}(\geq 191.8 v s<191.8)$ & 3.583(1.619-7.928) & 0.002 & - & - \\
\hline $\operatorname{HALP}(<32.4 v s \geq 32.4)$ & $0.254(.117-0.253)$ & 0.001 & $\begin{array}{l}0.352 \\
(0.154-0.804)\end{array}$ & 0.013 \\
\hline
\end{tabular}

HR, hazard ratio; CI: confidence interval; PSA, prostate-specific antigen; BMI, body mass index; pT-stage, pathological T staging; pN-stage, pathological lymph node status; ADT, androgen-deprivation therapy; ASA, American Society of Anesthesiologists; NLR: neutrophil-to-lymphocyte ratio; PLR:

platelet-to-lymphocyte ratio; HALP, hemoglobin, albumin, lymphocyte, and platelet score.

Table 4. Univariate and multivariate analysis of factors associated with PSA-PFS in oPCA patients, who had cytoreductive radical prostatectomy

\begin{tabular}{|c|c|c|c|c|}
\hline \multirow[t]{2}{*}{ Variable } & \multicolumn{2}{|l|}{ Univariate analysis } & \multicolumn{2}{|c|}{ Multivariate analysis } \\
\hline & HR $(95 \%$ CI) & $\begin{array}{l}\mathbf{P} \\
\text { value }\end{array}$ & HR (95\% CI) & $\begin{array}{l}\mathbf{P} \\
\text { value }\end{array}$ \\
\hline Age $(\geq 69 \mathrm{vs}<69)$ & $0.427(0.181-1.007)$ & 0.052 & & \\
\hline $\operatorname{PSA}(\geq 20 \mathrm{vs}<20)$ & $2.202(0.706-6.864)$ & 0.174 & & \\
\hline $\mathrm{BMI}(\geq 24 \mathrm{vs}<24)$ & $1.221(0.518-2.874)$ & 0.648 & & \\
\hline diabetes & $1.047(0.352-3.119)$ & 0.934 & & \\
\hline hypertension & $1.041(0.443-2.445)$ & 0.926 & & \\
\hline $\begin{array}{l}\text { Pathologic Gleason } \\
\text { Score }\end{array}$ & $2.371(1.060-5.303)$ & 0.036 & - & - \\
\hline pT34vs2 & $2.258(0.302-16.892)$ & 0.427 & & \\
\hline pN-stage & $1.385(0.596-3.215)$ & 0.449 & & \\
\hline Positive margins & $0.542(0.219-1.338)$ & 0.184 & & \\
\hline ASA grade $(1 \& 2$ vs $3 \& 4)$ & $0.915(0.475-1.761)$ & 0.789 & & \\
\hline Neoadjuvant ADT & $1.046(0.445-2.458)$ & 0.918 & & \\
\hline $\operatorname{NLR}(\geq 2.9 v s<2.9)$ & $2.402(1.027-5.617)$ & 0.043 & - & - \\
\hline $\operatorname{PLR}(\geq 191.8 v s<191.8)$ & 2.945(1.182-7.336) & 0.02 & - & - \\
\hline $\operatorname{HALP}(<32.4 v s \geq 32.4)$ & $0.275(0.116-0.653)$ & 0.003 & $\begin{array}{l}0.275 \\
(0.116-0.653)\end{array}$ & 0.003 \\
\hline
\end{tabular}

$\mathrm{HR}$, hazard ratio; CI: confidence interval; PSA, prostate-specific antigen; BMI, body mass index; pT-stage, pathological T staging; pN-stage, pathological lymph node status; ADT, androgen-deprivation therapy; ASA, American Society of Anesthesiologists; NLR: neutrophil-to-lymphocyte ratio; PLR:

platelet-to-lymphocyte ratio; HALP, hemoglobin, albumin, lymphocyte, and platelet score. 

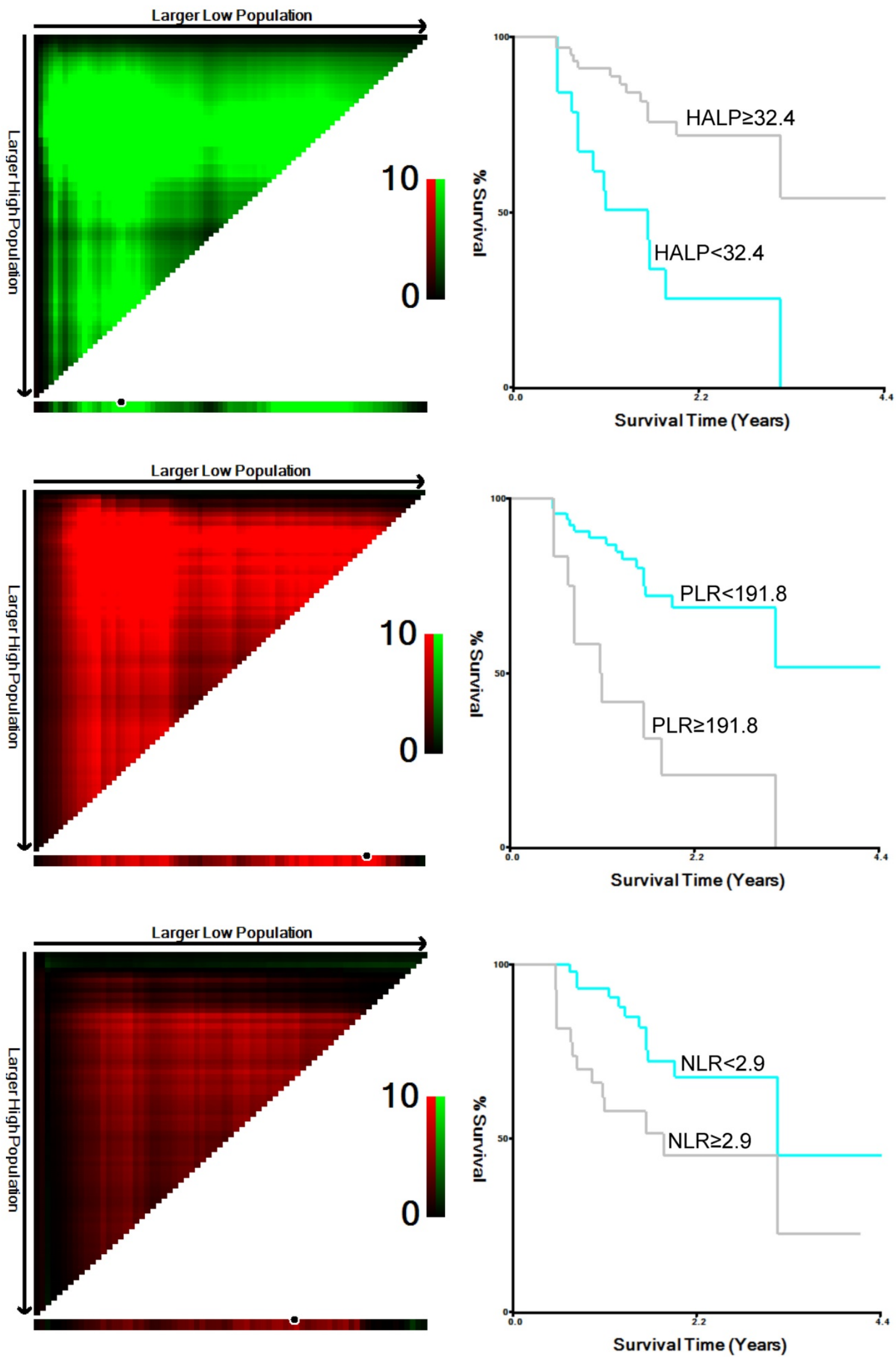

Figure 1. Cutoff values for HALP, PLR and NLR, as determined by X-tile software. 

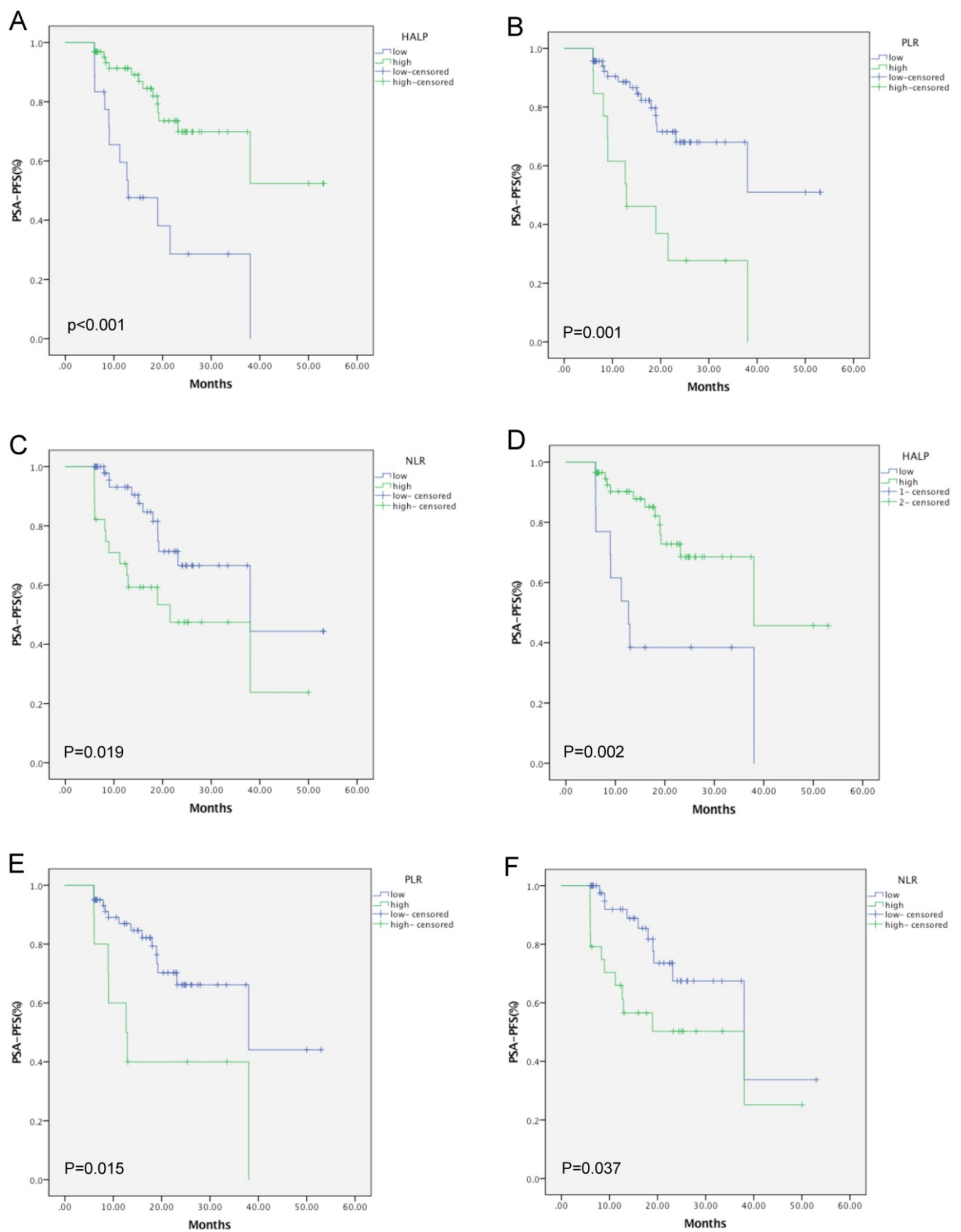

Figure 2. Kaplan-Meier curves for PSA-PFS in mPCA (A, B, C) and oPCA (D, E, F) patients according to the HALP score, PLR, and NLR, respectively. 

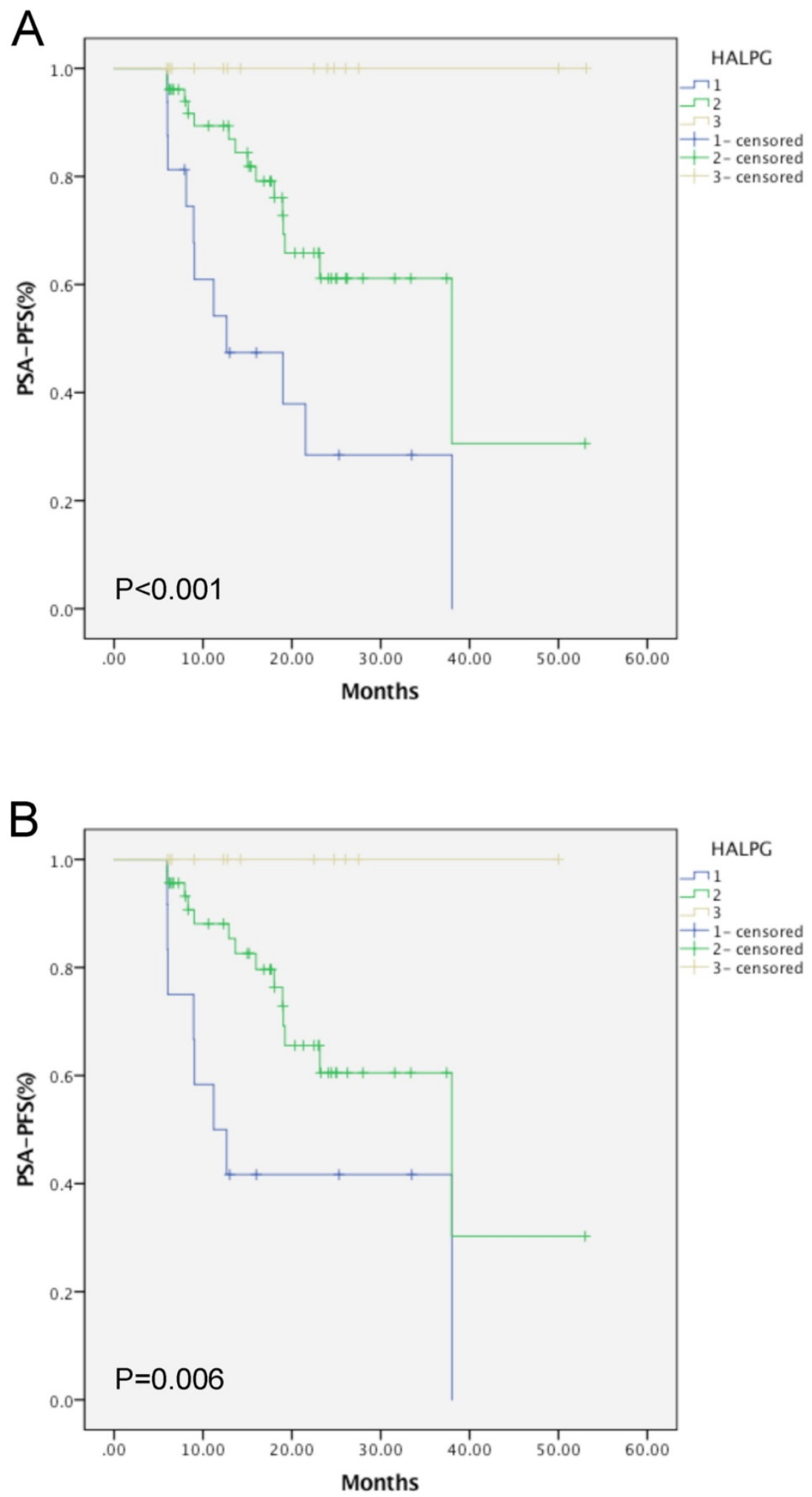

Figure 3. Kaplan-Meier curves of mPCA (A) and oPCA (B) patients based on the HALPG score.

\section{Creation of the HALPG value as a new prognostic model index}

Based on the multivariate analysis, PGS was identified as an important predictor, in addition to the HALP score. Thus, we combined PGS and the HALP score (HALPG) to construct a new index for prognostic prediction. The HALPG score is defined as follows: $\mathrm{HALPG}=1$ (HALP $<32.4$ and PGS $>7$; high risk), $\mathrm{HALPG}=2$ (HALP $<32.4$ and PGS $\leq 7$ or HALP $\geq 32.4$ and $\mathrm{PGS}>7$; intermediate risk), $\mathrm{HALPG}=3$ (HALP $\geq 32.4$ and $P G S \leq 7$; low risk). According to the 
HALPG score, 16 patients were classified into group 1,51 patients in group 2, and another 15 patients in group 3. Further univariate analysis based on the HALPG score demonstrated that a low HALPG score was a poor predictor of PSA-PFS prognosis based on the log-rank test (Figure 3). Multivariate analysis confirmed the HALPG score as an independent risk predictor for PSA-PFS in patients with $\mathrm{mPCA}$ or oPCA after CRP (HR = 0.270, 95\% CI: 0.138-0.529, P < $0.001, \mathrm{HR}=0.488,95 \% \mathrm{CI}: 0.314-0.759, \mathrm{P}=0.001)$ (Table 5). In addition, the discriminatory abilities of inflammation-based prognostic scores and clinical indices were also compared by AUC analysis for PSA-PFS in MPCA or oPCA patients (Figure 4). The AUC values for the HALPG score were 0.744 (95\% CI: $0.636-0.834)$ and 0.72 (95\% CI: 0.600-0.821) in mPCA and oPCA patients, respectively, and the AUC was the strongest factor among other indices such as HALP, PGS, NLR, PLR, positive margin (Table 7, Table 6, Table 8) for predicting recurrence in mPCA patients. Overall, our data demonstrated that the HALPG and HALP scores have higher prediction accuracy than other related indices in the PFS of mPCA and oPCA. However, the HALPG score appeared to be a better independent predictive factor than the HALP score for PFS in both MPCA and oPCA. Moreover, the HALPG score was also noticed to be better at risk stratification and predicting the prognosis of mPCA patients.

Table 5. Univariate and multivariate analysis of factors associated with PFS-PSA in mPCA patients who underwent cytoreductive radical prostatectomy

\begin{tabular}{|c|c|c|c|c|}
\hline \multirow[t]{2}{*}{ Variable } & \multicolumn{2}{|c|}{ Univariate analysis } & \multicolumn{2}{|c|}{ Multivariate analysis } \\
\hline & HR $(95 \%$ CI) & P value & HR $(95 \%$ CI) & P value \\
\hline Age $(\geq 69 v s<69)$ & $0.422(0.188-0.951)$ & 0.037 & - & - \\
\hline $\operatorname{PSA}(\geq 20 \mathrm{vs}<20)$ & $1.745(0.614-4.964)$ & 0.296 & & \\
\hline $\operatorname{BMI}(\geq 24 \mathrm{vs}<24)$ & $1.019(0.464-2.237)$ & 0.963 & & \\
\hline diabetes & $1.317(0.551-3.147)$ & 0.536 & & \\
\hline hypertension & $1.266(0.585-2.739)$ & 0.55 & & \\
\hline $\begin{array}{l}\text { Pathologic Gleason } \\
\text { Score }\end{array}$ & $2.401(1.290-4.469)$ & 0.006 & - & - \\
\hline pT34vs2 & $1.185(0.667-2.106)$ & 0.562 & & \\
\hline pN-stage & $1.461(0.671-3.181)$ & 0.34 & & \\
\hline Positive margins & $0.791(0.351-1.783)$ & 0.572 & & \\
\hline ASA grade $(1 \& 2$ vs $3 \& 4)$ & $1.233(0.494-3.078)$ & 0.653 & & \\
\hline Neoadjuvant ADT & $1.298(0.578-2.918)$ & 0.527 & & \\
\hline $\operatorname{NLR}(\geq 2.9 \mathrm{vs}<2.9)$ & $2.452(1.131-5.318)$ & 0.023 & - & - \\
\hline $\operatorname{PLR}(\geq 191.8 v s<191.8)$ & $3.583(1.619-7.928)$ & 0.002 & - & - \\
\hline $\operatorname{HALP}(<32.4 \mathrm{vs} \geq 32.4)$ & $0.254(.117-0.253)$ & 0.001 & - & - \\
\hline HLAPG & $0.270(0.138-0.529)$ & $<0.001$ & $\begin{array}{l}0.270 \\
(0.138-0.529)\end{array}$ & $<0.001$ \\
\hline
\end{tabular}

HR, hazard ratio; CI: confidence interval; PSA, prostate-specific antigen; BMI, body mass index; $\mathrm{pT}$-stage, pathological T staging; $\mathrm{pN}$-stage, pathological lymph node status; ADT, androgen-deprivation therapy; ASA, American Society of Anesthesiologists; NLR: neutrophil-to-lymphocyte ratio; PLR:

platelet-to-lymphocyte ratio; HALP, hemoglobin, albumin, lymphocyte, and platelet score; HALPG, combination of the pathologic Gleason score and the HALP score
Table 6. Univariate and multivariate analysis of factors associated with PFS-PSA in oPCA patients who underwent cytoreductive radical prostatectomy

\begin{tabular}{|c|c|c|c|c|}
\hline \multirow[t]{2}{*}{ Variable } & \multicolumn{2}{|l|}{ Univariate analysis } & \multicolumn{2}{|c|}{ Multivariate analysis } \\
\hline & HR $(95 \%$ CI) & Pvalue & HR $(95 \% \mathrm{CI})$ & P value \\
\hline Age $(\geq 69 \mathrm{vs}<69)$ & $0.427(0.181-1.007)$ & 0.052 & & \\
\hline $\operatorname{PSA}(\geq 20 \mathrm{vs}<20)$ & $2.202(0.706-6.864)$ & 0.174 & & \\
\hline $\operatorname{BMI}(\geq 24 v s<24)$ & $1.221(0.518-2.874)$ & 0.648 & & \\
\hline diabetes & $1.047(0.352-3.119)$ & 0.934 & & \\
\hline hypertension & $1.041(0.443-2.445)$ & 0.926 & & \\
\hline $\begin{array}{l}\text { Pathologic Gleason } \\
\text { Score }\end{array}$ & $2.371(1.060-5.303)$ & 0.036 & - & - \\
\hline pT34vs2 & $2.258(0.302-16.892)$ & 0.427 & & \\
\hline pN-stage & $1.385(0.596-3.215)$ & 0.449 & & \\
\hline Positive margins & $0.542(0.219-1.338)$ & 0.184 & & \\
\hline $\begin{array}{l}\text { ASA grade }(1 \& 2 \text { vs } \\
3 \& 4)\end{array}$ & $0.915(0.475-1.761)$ & 0.789 & & \\
\hline Neoadjuvant ADT & $1.046(0.445-2.458)$ & 0.918 & & \\
\hline $\operatorname{NLR}(\geq 2.9 \mathrm{vs}<2.9)$ & 2.402(1.027-5.617) & 0.043 & - & - \\
\hline $\operatorname{PLR}(\geq 191.8 v s<191.8)$ & $2.945(1.182-7.336)$ & 0.02 & - & - \\
\hline $\operatorname{HALP}(<32.4 v s \geq 32.4)$ & $0.275(0.116-0.653)$ & 0.003 & - & - \\
\hline HLAPG & $0.302(0.142-0.640)$ & 0.002 & $\begin{array}{l}0.488 \\
(0.314-0.759)\end{array}$ & 0.001 \\
\hline
\end{tabular}

HR, hazard ratio; CI: confidence interval; PSA, prostate-specific antigen; BMI, body mass index; pT-stage, pathological T staging; pN-stage, pathological lymph node status; ADT, androgen-deprivation therapy; ASA, American Society of Anesthesiologists; NLR: neutrophil-to-lymphocyte ratio; PLR: platelet-to-lymphocyte ratio; HALP, hemoglobin, albumin, lymphocyte, and platelet score; HALPG, combination of the pathologic Gleason score and the HALP score.

Table 7. AUC analysis of mPCA patients for various indices

\begin{tabular}{llll}
\hline Variable & AUC & SE & 95\% CI \\
\hline HALPG & 0.744 & 0.0458 & $0.636-0.834$ \\
HALP & 0.677 & 0.054 & $0.565-0.776$ \\
PLR & 0.666 & 0.051 & $0.553-0.766$ \\
NLR & 0.644 & 0.0578 & $0.531-0.747$ \\
Gleason_Score & 0.688 & 0.053 & $0.576-0.786$ \\
Age & 0.565 & 0.0597 & $0.451-0.674$ \\
\hline
\end{tabular}

AUC, area under the curve; SE, standard error; NLR: neutrophil-to-lymphocyte ratio; PLR: platelet-to-lymphocyte ratio; HALP, hemoglobin, albumin, lymphocyte, and platelet score; HALPG, combination of the pathologic Gleason score and the HALP score.

Table 8. AUC analysis of oPCA patients for various indices

\begin{tabular}{llll}
\hline Variable & AUC & SE & $\mathbf{9 5 \% ~ C I ~}$ \\
\hline HALPG & 0.72 & 0.05 & $0.600-0.821$ \\
HALP & 0.663 & 0.0573 & $0.540-0.772$ \\
PLR & 0.628 & 0.0538 & $0.504-0.740$ \\
NLR & 0.648 & 0.0628 & $0.524-0.758$ \\
Gleason_Score & 0.661 & 0.058 & $0.538-0.770$ \\
Age & 0.592 & 0.0633 & $0.468-0.708$
\end{tabular}

AUC, area under the curve; SE, standard error; NLR: neutrophil-to-lymphocyte ratio; PLR: platelet-to-lymphocyte ratio; HALP, hemoglobin, albumin, lymphocyte, and platelet score; HALPG, combination of the pathologic Gleason score and the HALP score.

\section{Discussion}

In this study, we assessed the ability of the novel index HALP in mPCA. It was observed to be not only significantly associated with the MPCA prognosis but also with some other clinicopathological features such as PGS, ASA, NLR, and PLR. More specifically, a low HALP score showed a significant association with 
tumor progression and appeared to be an unfavorable risk factor for both the mPCA and oPCA patient subgroups. Furthermore, the combination of PGS and the HALP score enabled us to create a new prognostic index, HALPG, which was observed to be an independent risk factor for PSA-PFS in $\mathrm{mPCA}$ patients. Consequently, we hypothesized that the predictive significance of the HALPG score would help clinicians to identify high-risk patients in a timely manner with more accuracy as well as to provide reasonable treatment options, postoperatively.
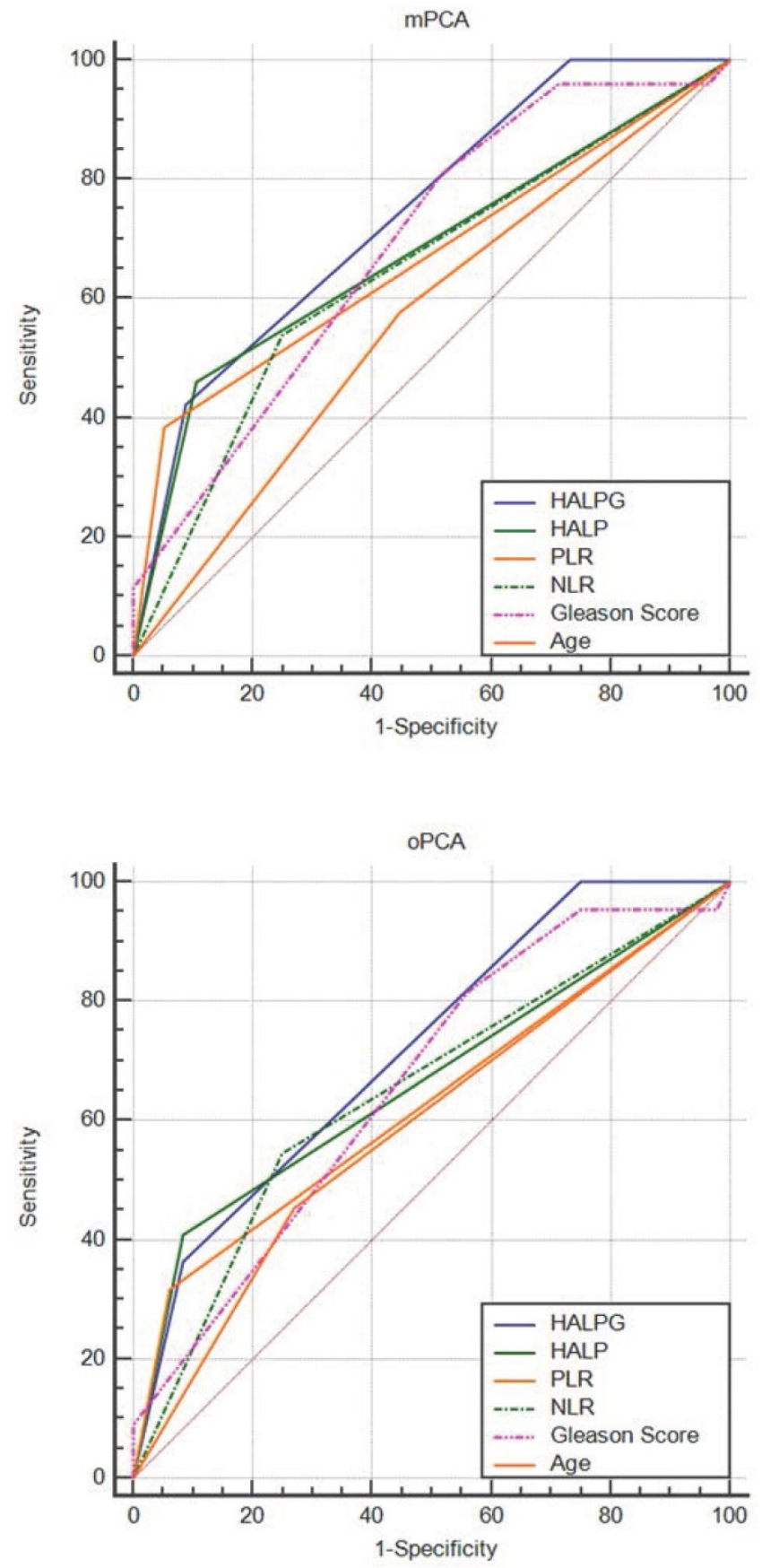

Figure 4. Comparison of the predictive ability of the HALPG score and other clinical parameters by ROC curve analysis in MPCA and oPCA patients.
The role of nutrition and immunity in predicting the prognosis of cancer patients has recently gained attention [26, 27]. The tumor itself seems to be a chronic consumption disease, especially advanced tumors, and the level of hemoglobin has been observed to be significantly related to survival and tumor progression in cancer patients [28]. In their systematic review, Caro et. al. [20] emphasized anemia as being an independent prognostic factor for cancer patients and identified that the risk of death associated with anemia in prostate cancer patients increased by $47 \%$. Moreover, the serum albumin level acts as a simple surrogate for assessing protein levels and is an indicator of the nutritional status. The literature supports the hypothesis that the serum albumin level is significantly correlated with cancer survival [19, 29]. Furthermore, Sejima et. al. [30] have demonstrated that preoperative hypoalbuminemia may lead to the spread of localized prostate cancer and be associated with biochemical recurrence. In addition, other studies have reported that the tumor microenvironment is mainly regulated by inflammatory cells, which indispensably contribute to tumor progression $[12,31]$. For instance, platelets have been shown to interact with tumor cells and promote their survival and metastasis through different mechanisms. By accumulating platelets, tumor cells can escape the human immune system. Other studies have indicated that platelets protect tumors from tumor necrosis factor- $\alpha$ (TNF- $\alpha$ )- mediated cytotoxicity and the high shear forces that may potentially damage them in flowing blood [32, 33]. Additionally, lymphopenia, which is quite common in patients with advanced cancer, is an independent prognostic factor for overall and progression-free survival in cancer patients [34], probably because by releasing certain cytokines, like interferon- $\gamma$ and TNF- $\alpha$, lymphocytes can effectively control tumors and improve the prognosis of patients with different cancers [11]. Based on these different observations, it was evident that hemoglobin, albumin, and lymphocytes can be favorable risk factors; while platelets may be an unfavorable risk factor. In this context, our results showed that the HALP score is significantly associated with mPCA prognosis and that a low HALP score predicts a poor clinical outcome.

In the present study, besides the HALP score, we also examined the predictive value of other indices like NLR and PLR, which have been widely studied in prostate cancer patients. Previous studies have reported in detail that high NLR and PLR values are significantly associated with a poor prognosis in prostate cancer patients $[15,16]$. However, by multivariate analysis, our study identified that both of 
these two indices had no statistically significant association and that the predictive ability of the HALP score was indeed higher than those of NLR and PLR. The significant association of the PGS with the biochemical progression and long-term survival of prostate cancer patients also has been reported [35]. Consistent with these findings, we also observed that a high PGS is an independent risk factor for a decreased PSA-PFS. Thus, by combining the PGS with the HALP score, we created a new index, HALPG, which expectedly showed a better prognostic significance in PSA-PFS than either the HALP score or PGS alone. This result led us to hypothesize that a low HALPG score may not only be negatively correlated with the prognosis of mPCA or oPCA after cRP, but it is also better than the other indices reported previously. Importantly, more recently, some new techniques such as genetic testing and fluid biopsy have started to emerge. These methods appear to improve the accuracy of mPCA prognosis, but they have some disadvantages such as a relatively high cost and time-consuming analysis; moreover, most of them are still in clinical trials. Therefore, as a fast, accurate, and low-cost biomarker, the HALPG score can help us to predict postoperative recurrence and to make conclusive decisions on the precise treatment option for mPCA patients, including the choice of adjuvant therapy and follow-up arrangements. According to our analysis, the low HALPG score group had the worst outcome. Thus, in this group of patients, more powerful adjuvant therapy can be used to prevent postoperative recurrence, leading to a prolonged survival. Also, these patients at high risk for cancer recurrence should be followed more frequently after surgery to select the appropriate treatment option.

However, it is important to recognize some limitations of our study. First, as our study is retrospective in nature and the data are from patients treated with a single treatment, our results have the potential to be biased in terms of the population choice. Second, our data sample is small. Third, the follow-up time was also relatively short. Thus, these limitations can potentially limit the accuracy of our results. Therefore, the ability and accuracy of the HALP and HALPG scores in predicting the prognosis of mPCA patients should be further validated by including additional prospective, multicenter studies.

\section{Conclusion}

In summary, our results suggest that the preoperative HALP score can be a valid prognostic index for patients with mPCA. In addition, the combination of the PGS and the HALP score as a new index, HALPG, seems to be an independent risk factor for PSA-PFS in mPCA patients. Similarly, both of these indices also can be applied in oPCA patients. Finally, these indices have the ability to stratify risk and guide treatment options for postoperative mPCA patients.

\section{Acknowledgements}

This work was supported by the National Natural Science Foundation of China (grant number: 81472389)

\section{Competing Interests}

The authors have declared that no competing interest exists.

\section{References}

1. Siegel RL, Miller KD, Jemal A. Cancer statistics, 2018. CA Cancer J Clin. 2018; 68: 7-30.

2. Fizazi K, Tran N, Fein L, Matsubara N, Rodriguez-Antolin A, Alekseev B, et al. Abiraterone plus Prednisone in Metastatic, Castration-Sensitive Prostate Cancer. N Engl J Med. 2017; 377: 352-60.

3. Gillessen S, Attard G, Beer T, Beltran H, Bossi A, Bristow R, et al. Management of Patients with Advanced Prostate Cancer: The Report of the Advanced Prostate Cancer Consensus Conference APCCC 2017. Eur Urol. 2018; 73: 178-211.

4. Mathieu R, Korn SM, Bensalah K, Kramer G, Shariat SF. Cytoreductive radical prostatectomy in metastatic prostate cancer: Does it really make sense? World J Urol. 2017; 35: 567-77.

5. Hellman S, Weichselbaum R. Oligometastases. J Clin Oncol. 1995; 13: 8-10.

6. Singh D, Yi WS, Brasacchio RA, Muhs AG, Smudzin T, Williams JP, et al. Is there a favorable subset of patients with prostate cancer who develop oligometastases? International Journal of Radiation Oncology*Biology*Physics. 2004; 58: 3-10.

7. James N, Sydes M, Clarke N, Mason M, Dearnaley D, Spears M, et al. Addition of docetaxel, zoledronic acid, or both to first-line long-term hormone therapy in prostate cancer (STAMPEDE): survival results from an adaptive, multiarm, multistage, platform randomised controlled trial. Lancet. 2016; 387: 1163-77.

8. Miller KD, Siegel RL, Lin CC, Mariotto AB, Kramer JL, Rowland JH, et al. Cancer treatment and survivorship statistics, 2016. CA Cancer J Clin. 2016; 66: 271-89.

9. McMillan DC. Systemic inflammation, nutritional status and survival in patients with cancer. Curr Opin Clin Nutr Metab Care. 2009; 12: 223-6.

10. Bindea G, Mlecnik B, Fridman WH, Pages F, Galon J. Natural immunity to cancer in humans. Curr Opin Immunol. 2010; 22: 215-22.

11. Mantovani A, Allavena P, Sica A, Balkwill F. Cancer-related inflammation. Nature. 2008; 454: 436-44.

12. Coussens L, Werb Z. Inflammation and cancer. Nature. 2002; 420: 860-7.

13. Coffelt $S$, de Visser $K$. Cancer: Inflammation lights the way to metastasis. Nature. 2014; 507: 48-9.

14. Fan L, Wang R, Chi C, Cai W, Zhang Y, Qian H, et al. Systemic immune-inflammation index predicts the combined clinical outcome after sequential therapy with abiraterone and docetaxel for metastatic castration-resistant prostate cancer patients. Prostate. 2018; 78: 250-6.

15. Sidaway P. Prostate cancer: Platelet-to-lymphocyte ratio predicts prostate cancer prognosis. Nat Rev Urol. 2015; 12: 238.

16. Lorente D, Mateo J, Templeton A, Zafeiriou Z Bianchini D, Ferraldeschi R, et al. Baseline neutrophil-lymphocyte ratio (NLR) is associated with survival and response to treatment with second-line chemotherapy for advanced prostate cancer independent of baseline steroid use. Ann Oncol. 2015; 26: 750-5.

17. Rock CL, Doyle C, Demark-Wahnefried W, Meyerhardt J, Courneya KS, Schwartz AL, et al. Nutrition and physical activity guidelines for cancer survivors. CA Cancer J Clin. 2012; 62: 243-74.

18. Fan L, Wang X, Chi C, Wang Y, Cai W, Shao X, et al. Prognostic nutritional index predicts initial response to treatment and prognosis in metastatic castration-resistant prostate cancer patients treated with abiraterone. Prostate. 2017; 77: 1233-41.

19. Liu X, Meng QH, Ye Y, Hildebrandt MA, Gu J, Wu X. Prognostic significance of pretreatment serum levels of albumin, $\mathrm{LDH}$ and total bilirubin in patients with non-metastatic breast cancer. Carcinogenesis. 2015; 36: 243-8.

20. Caro J, Salas M, Ward A, Goss G. Anemia as an independent prognostic factor for survival in patients with cancer: a systemic, quantitative review. Cancer. 2001; 91: 2214-21.

21. Peng $\mathrm{D}$, Zhang $\mathrm{C}$, Tang $\mathrm{O}$ Zhang $\mathrm{L}$, Yang $\mathrm{K}, \mathrm{Yu}, \mathrm{X}$ et al Prognostic significance of the combination of preoperative hemoglobin and albumin levels and lymphocyte and platelet counts (HALP) in patients with renal cell carcinoma after nephrectomy. BMC Urol. 2018; 18: 20. 
22. Peng D, Zhang C, Gong Y, Hao H, Guan B, Li X, et al. Prognostic significance of HALP (hemoglobin, albumin, lymphocyte and platelet) in patients with bladder cancer after radical cystectomy. Sci Rep. 2018; 8: 794.

23. Chen X, Xue L, Wang W, Chen H, Zhang W, Liu K, et al. Prognostic significance of the combination of preoperative hemoglobin, albumin, lymphocyte and platelet in patients with gastric carcinoma: a retrospective cohort study. Oncotarget. 2015; 6: 41370-82.

24. Jiang H, Li H, Li A, Tang E, Xu D, Chen Y, et al. Preoperative combined hemoglobin, albumin, lymphocyte and platelet levels predict survival in patients with locally advanced colorectal cancer. Oncotarget. 2016; 7: 72076-83.

25. Camp R, Dolled-Filhart M, Rimm D. X-tile: a new bio-informatics tool for biomarker assessment and outcome-based cut-point optimization. Clin Cancer Res. 2004; 10: 7252-9.

26. Ryan A Power D, Daly $\mathrm{L}$ Cushen $\mathrm{S}$, Ní Bhuachalla $\overline{\mathrm{E}}$, Prado $\mathrm{C}$ Cancer-associated malnutrition, cachexia and sarcopenia: the skeleton in the hospital closet 40 years later. Proc Nutr Soc. 2016; 75: 199-211.

27. Efremova M, Rieder D, Klepsch V, Charoentong P, Finotello F, Hackl H, et al. Targeting immune checkpoints potentiates immunoediting and changes the dynamics of tumor evolution. Nat Commun. 2018; 9: 32.

28. Belcher D, Ju J, Baek J, Yalamanoglu A, Buehler P, Gilkes D, et al. The quaternary state of polymerized human hemoglobin regulates oxygenation of breast cancer solid tumors: A theoretical and experimental study. PLoS ONE. 2018; 13: e0191275.

29. Ayhan A, Günakan E, Alyazıcı İ, Haberal N, Altunda ğ Ö, Dursun P. The preoperative albumin level is an independent prognostic factor for optimally debulked epithelial ovarian cancer. Arch Gynecol Obstet. 2017; 296: 989-95.

30. Sejima T, Iwamoto H, Masago T, Morizane S, Yao A, Isoyama T, et al. Low pre-operative levels of serum albumin predict lymph node metastases and ultimately correlate with a biochemical recurrence of prostate cancer in radical prostatectomy patients. Cent European J Urol. 2013; 66: 126-32.

31. Shiao S, Chu G, Chung L. Regulation of prostate cancer progression by the tumor microenvironment. Cancer Lett. 2016; 380: 340-8.

32. Jurasz P, Alonso-Escolano D, Radomski MW. Platelet--cancer interactions: mechanisms and pharmacology of tumour cell-induced platelet aggregation. Br J Pharmacol. 2004; 143: 819-26.

33. Schumacher D, Strilic B, Sivaraj K, Wettschureck N, Offermanns S. Platelet-derived nucleotides promote tumor-cell transendothelial migration and metastasis via P2Y2 receptor. Cancer Cell. 2013; 24: $130-7$.

34. Ray-Coquard I, Cropet C, Van Glabbeke M, Sebban C, Le Cesne A, Judson I, et al. Lymphopenia as a prognostic factor for overall survival in advanced carcinomas, sarcomas, and lymphomas. Cancer Res. 2009; 69: 5383-91.

35. Andrén O, Fall K, Franzén L, Andersson S, Johansson J, Rubin M. How well does the Gleason score predict prostate cancer death? A 20-year followup of a population based cohort in Sweden. J Urol. 2006; 175: 1337-40. 